\begin{tabular}{|c|c|c|}
\cline { 2 - 3 } & REVISTA SABERES APUDEP & Volumen 4 Número 1 \\
\hline
\end{tabular}

\title{
ÁREAS NATURALES EN ZONAS COSTERAS, ESPACIOS EN RIESGO POR DISPOSICIÓN FINAL DE RESIDUOS SÓLIDOS DE LA COMUNIDAD DE GOBEA
}

\author{
NATURAL AREAS IN COASTAL ZONE, LOCATIONS AT RISK DUE TO FINAL \\ DISPOSAL OF SOLID WASTE FROM GOBEA COMMUNITY.
}

\author{
Vera De La Cruz Cabrera
}

Universidad de Panamá. Centro Regional Universitario de Colón. Panamá. vera.delacruz@up.ac.pa https://orcid.org/ 0000-0003-3718-4299

Reyna Cantón

Universidad de Panamá. Centro Regional Universitario de Colón. Panamá. reynacanton@hotmail.com https://orcid.org/ 0000-0001-6121-0835

\section{Yireika Garibaldi}

Universidad de Panamá. Centro Regional Universitario de Colón. Panamá.yirethg24@gmail.com https://orcid.org/ 0000-0002-7941-039X

\section{Viodelda Ortega}

Universidad de Panamá. Centro Regional Universitario de Colón. Panamá. ortegaviodelda@gmail.com https://orcid.org/0000-0002-7357-7893

\section{RESUMEN}

El manejo de los residuos sólidos se ha convertido en un problema mundial de tipo ambiental y es parte indisoluble de las actividades humanas. Su incremento, su manejo y disposición final es un asunto pendiente por resolver en Panamá debido a los impactos negativos al ambiente y el deterioro acelerado de los ecosistemas, afectando el agua, el aire, el suelo y el desplazamiento de animales en busca de nuevos hábitats naturales. A finales del siglo XX, el vínculo hombre-naturaleza se fortalece con el uso de recursos naturales produciendo más residuos sin una gestión sostenible, acentuando problemas ambientales como el cambio climático, generando olores ofensivos por la descomposición de la materia y su arrastre por aguas subterráneas. El estudio se desarrolla en la comunidad de Gobea en Costa Abajo, Provincia de Colón, con el propósito de mostrar las áreas naturales de la franja costera usadas para disposición final de residuos sólidos, la caracterización de éstos y resaltar los posibles impactos negativos que se generan al ambiente. 


\begin{tabular}{|c|c|c|}
\cline { 2 - 3 } & REVISTA SABERES APUDEP & Volumen 4 Número 1 \\
\hline
\end{tabular}

La metodología desarrollada presenta una fase de observación directa y tres momentos de trabajo de campo donde se indicaron las áreas afectadas, se clasificaron y describieron los diversos residuos encontrados y registraron sus datos. Los resultados evidenciaron puntos críticos como vertederos improvisados de residuos sólidos posiblemente arrastrados por el oleaje hacia el mar, lo que demuestra la necesidad de estrategias ambientales para conservar estas zonas costeras como espacios de obtención de recursos alimenticios para la comunidad.

PALABRAS CLAVE: residuos sólidos, zona costera, riesgo ambiental, sensibilización Ambiental

\section{ABSTRACT}

Solid waste management has become a global environmental problem and it is an inseparable part of human activities. Its increase, management and final disposal is a pending issue to be solved in Panama due to the negative impacts on the environment and the accelerated deterioration of ecosystems, affecting water, air, soil and the movement of animals in search of new natural habitats. At the end of the 20th century, the man-nature link was strengthened with the use of natural resources, producing more waste without sustainable management, emphasizing environmental problems such as climate change, generating offensive odors due to the decomposition of elements and its drag through groundwater. The descriptive study is developed in the community of Gobea in Costa Abajo, Province of Colón, with the purpose of showing the natural areas of the coastal strip used for final disposal of solid waste, characterizing these and highlighting the possible negative impacts that are generated to the environment. The methodology developed presents a direct observation phase and three moments of field work where the affected areas were indicated, the various residues found were classified and described, and their data recorded. The results showed critical points such as improvised solid waste dumps possibly carried by the waves towards the sea, which shows the need for environmental strategies to conserve these coastal areas as spaces for obtaining food resources for the community.

KEYWORDS: solid waste, coastal zone, environmental risk, environmental awareness 


\section{INTRODUCCIÓN}

La creciente disponibilidad de residuos sólidos se vincula con el incremento de la densidad poblacional y de la producción industrial, donde la tendencia de generación de nuevas versiones de productos, incrementan el descarte de éstos-en un corto plazo, convirtiendo algunos espacios en vertederos improvisados. Aunque su disposición final debe ser en el vertedero, vemos con preocupación cómo son arrastrados por grandes precipitaciones pluviales o vientos, dispersándola hasta llegar a lugares no apropiados. (Ojeda \& Quintero 2008, Rodríguez 2008, Esquer 2009, Gázquez 2010, Saéz \& Urdaneta 2014).

Las áreas naturales en zonas costeras son espacios multifuncionales que ofrecen un amplio rango de paisajes, usos y actividades. Dentro de estos espacios, las playas se constituyen en uno de los atractivos más importantes debido a su valor ecológico, productivo y estético; sin embargo, está bajo amenaza por todos los residuos sólidos que se perpetúan en la arena (Hurtado, 2010). En la actualidad se tiene poca información del estado de las playas y ríos de las comunidades de la Costa Abajo, situadas en el Caribe de la Provincia de Colón, (Autoridad Nacional del Ambiente, 2012).

La disposición de residuos sólidos en las playas es un aspecto a considerar, siendo necesario el desarrollo de programas de corte ambiental en las comunidades que conforman las costas del Caribe. La gestión deficiente de la basura, provoca que cada año 8 millones de toneladas de desechos plásticos lleguen a las costas y luego al mar abierto (Banco Mundial, 2018). El plástico abunda en todos los océanos y en las profundidades en su forma original o de micro plásticos. Los aparejos de pesca abandonados, representan un importante porcentaje de los desperdicios arrojados al mar causan una reducción de poblaciones de peces, y las redes fantasmas representan el 10\% de plásticos depositados en el mar (Macfadyen et al, 2011).

La mala disposición de los residuos es importante, por las connotaciones ambientales y socio culturales que pueden generarse para toda la población (Contreras 2008, Universidad de Panamá 2010, Alonzo \& Paz 2014, Saéz \& Urdaneta 2014). Los residuos sólidos depositados en las playas son el resultado de todo el descarte del hombre, por algo que no le encuentra valor alguno después de su primer uso. Aunque las autoridades locales deben estar a cargo de la aseo y ornato de la comunidad, es la conducta ambiental responsable de sus miembros 


\section{REVISTA SABERES APUDEP \\ ISSN L 2644-3805}

Acceso Abierto. Disponible en:

https://revistas.up.ac.pa/index.php/saberes_apudep

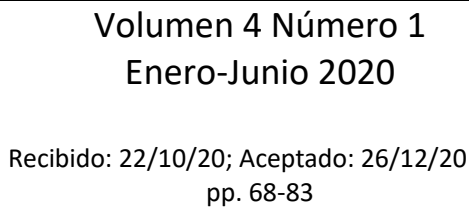

y visitantes los que deben ser el primer grupo preocupado para reducir la generación de residuos sólidos (Pereira \& Conto, 2008). Es necesario hacer énfasis en que la acumulación de estos representa un impacto negativo al medioambiente, sirve de fuente de contaminación, degrada el paisaje natural, prolifera la presencia de vectores de enfermedades, inclusive desmejora la calidad sanitaria de la arena ya que se convierte en medio para el desarrollo de microorganismos patógenos contaminando el agua. Adicionalmente, se debe asegurar que los residuos no se acumulen hasta llegar a ser desagradables o peligrosos (Valencia 2009, FEE 2010). Por otra parte, además de exponer la cantidad de residuos, es de igual importancia saber cuáles son los tipos de residuos sólidos más comunes en una playa pues, a partir de esta información, se pueden tomar medidas para prevenir o mitigar los impactos ambientales que los residuos generan. El término manejo de desechos se usa para designar el control humano de recolección, tratamiento y eliminación de los diferentes tipos de residuos. (Silgado 2006, Mazzeo 2012, Saéz \& Urdaneta 2014).

Diversos problemas son causados en las comunidades cercanas a los ríos y playas por la disposición de los residuos. Uno de esos problemas son la mala disposición de los residuos sólidos en las playas, lo que supone una falta de cultura ambiental por parte de los moradores. Para Junco y Rodríguez (2000), la generación de residuos y su mala disposición final afecta la calidad de vida del hombre y deteriora el ambiente. La actividad que cada ser humano realiza conforma su huella ecológica, o sea el impacto que su modo de vida tiene sobre los recursos naturales y el ambiente.

En la República de Panamá, los recuentos de basura, organizados por grupos ambientalistas, estudiantiles, y otros, demuestran la necesidad de concientizar a las comunidades costeras, a los municipios y departamentos de sanidad, principalmente las comunidades en Costa Abajo Provincia de Colón a atender las amenazas contra el medioambiente. Los riesgos en el manejo de los residuos sólidos pueden darse desde el lugar en el cual se generan, donde se acopian hasta su disposición final.

La versión XXIII de la gran limpieza de playas, costas y ríos realizada en septiembre de 2014; en 50 puntos del Istmo de Panamá; demuestra que se necesita poner mucha atención al problema de los desechos sólidos en sectores específicos como lo son las playas y costas del 


\section{REVISTA SABERES APUDEP \\ ISSN L 2644-3805}

Acceso Abierto. Disponible en:

https://revistas.up.ac.pa/index.php/saberes_apudep

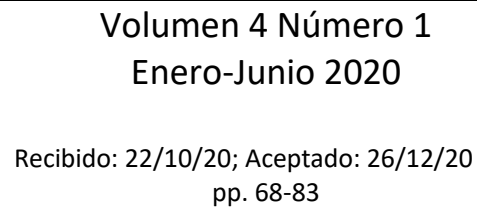

Recibido: 22/10/20; Aceptado: 26/12/20 pp. 68-83

país. Se recolectaron 36 toneladas de residuos sólidos, con aproximadamente un $75 \%$ d plásticos incluidos (Arias, 2015).

La franja costera de la comunidad de Gobea, es afectada por condiciones que contribuyen a su deterioro, tales como olores ofensivos producto de la acumulación y descomposición de residuos sólidos como resultado de actividades de la comunidad (Vallester \& Núñez 2007, Márquez Gulloso \& Rosado Vega 2011). Los malos olores pueden estar relacionados con las características de la zona del mar, siendo los olores de mayor énfasis animales en descomposición, residuos orgánicos e inorgánicos posiblemente debido a la ausencia de medidas de control y de conciencia ambiental por los usuarios de las playas y los moradores del lugar, siendo la temporada lluviosa la de mayor cantidad de residuos sólidos, posiblemente por las acciones derivadas de las actividades domésticas, turismo u otros.

Un aspecto interesante es la manifestación de fenómenos naturales y antrópicos que influyen en la extensión de la zona de la playa, lo que genera una acción de inclusión del área del vertedero improvisado y el área del cementerio de la comunidad (Blais et al 2010, Benseny, 2011). A simple vista se observa la mala disposición de los residuos sólidos en la playa de Gobea, dando una vista negativa del paisaje de la comunidad.

Según la situación actual los residuos sólidos generados y su mala disposición final, así como la necesidad de contar con investigaciones más recientes útiles para abordar el tema, se ha desarrollado el presente estudio de carácter descriptivo, estableciendo propósitos de indagar los sitios donde se encuentran en mala disposición sin un tratamiento apropiado y con la posibilidad de atentar contra la calidad ambiental del lugar, ya que se convierten en vertederos improvisados y colindan con la playa y el cementerio del lugar, lo que puede empeorar la situación. Por su naturaleza, estos residuos pasan por una degradación muy lenta o no pasan por ese proceso, lo que supone una posible condición de contaminación ambiental (Kofalusi \& Aguilar, 2006). Esta situación se agrava cuando los pobladores utilizan técnicas rudimentarias como la quema, uso de fosas improvisadas, entre otras para la eliminación permanente de todo residuo. 


\section{REVISTA SABERES APUDEP \\ ISSN L 2644-3805}

Acceso Abierto. Disponible en:

https://revistas.up.ac.pa/index.php/saberes_apudep

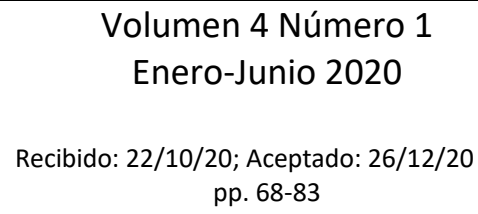

El estudio incluye la identificación de los diversos tipos de residuos, y elaborar un informe escrito registrando los datos obtenidos para que sirvan para establecer estrategias ambientales que ofrezcan un mejor manejo ya sea de separación de los que se degradan, como aquellos que puedan servir para actividad de aprovechamiento o reciclaje, cumpliendo con las normas que establecen. Por tal razón, se pretende exponer la necesidad inmediata de una gestión para minimizar la acumulación de residuos sólidos generados en las áreas naturales por su valor turístico, ambiental, económico y productivo. Pocos han sido los estudios que se han realizado sobre el tema, sin embargo; existe la necesidad de dar a conocer con certeza científica, información que puede ser útil y permita a las autoridades locales evaluar y considerar en la toma de decisiones una propuesta integral que vaya dirigida a lograr un mejor manejo de los residuos sólidos generados con alternativas útiles para ese escenario, dando atención a esta situación tan preocupante.

\section{MATERIALES Y METODOS}

\section{ÁREA DE ESTUDIO}

La comunidad de Gobea, se encuentra localizada en la Costa Abajo, Provincia de Colón situada en las siguientes coordenadas 9 $10^{\prime} 07^{\prime \prime} \mathrm{N}, 80^{\circ} 14^{\prime} 56^{\prime \prime}$ O. Elevación $32 \mathrm{~m}$, sobre el nivel del mar (Figura 1). La playa de la comunidad de Gobea, tiene una extensión 2.8 kilómetros, bordeada de bosque tropical. Presenta un clima tropical húmedo, donde la temperatura media anual está entre los $24^{\circ}$ a $30^{\circ}$ centígrados, una humedad relativa del $85 \%$ y precipitación anual de $3400 \mathrm{~mm}$. Las precipitaciones no desaparecen por completo porque llueve unos $65 \mathrm{~mm}$ al mes, y evidencia una temporada seca entre los meses de enero a abril y una lluviosa entre mayo a diciembre. 


\begin{tabular}{|c|c|c|}
\cline { 2 - 3 } & REVISTA SABERES APUDEP & Volumen 4 Número 1 \\
\hline
\end{tabular}

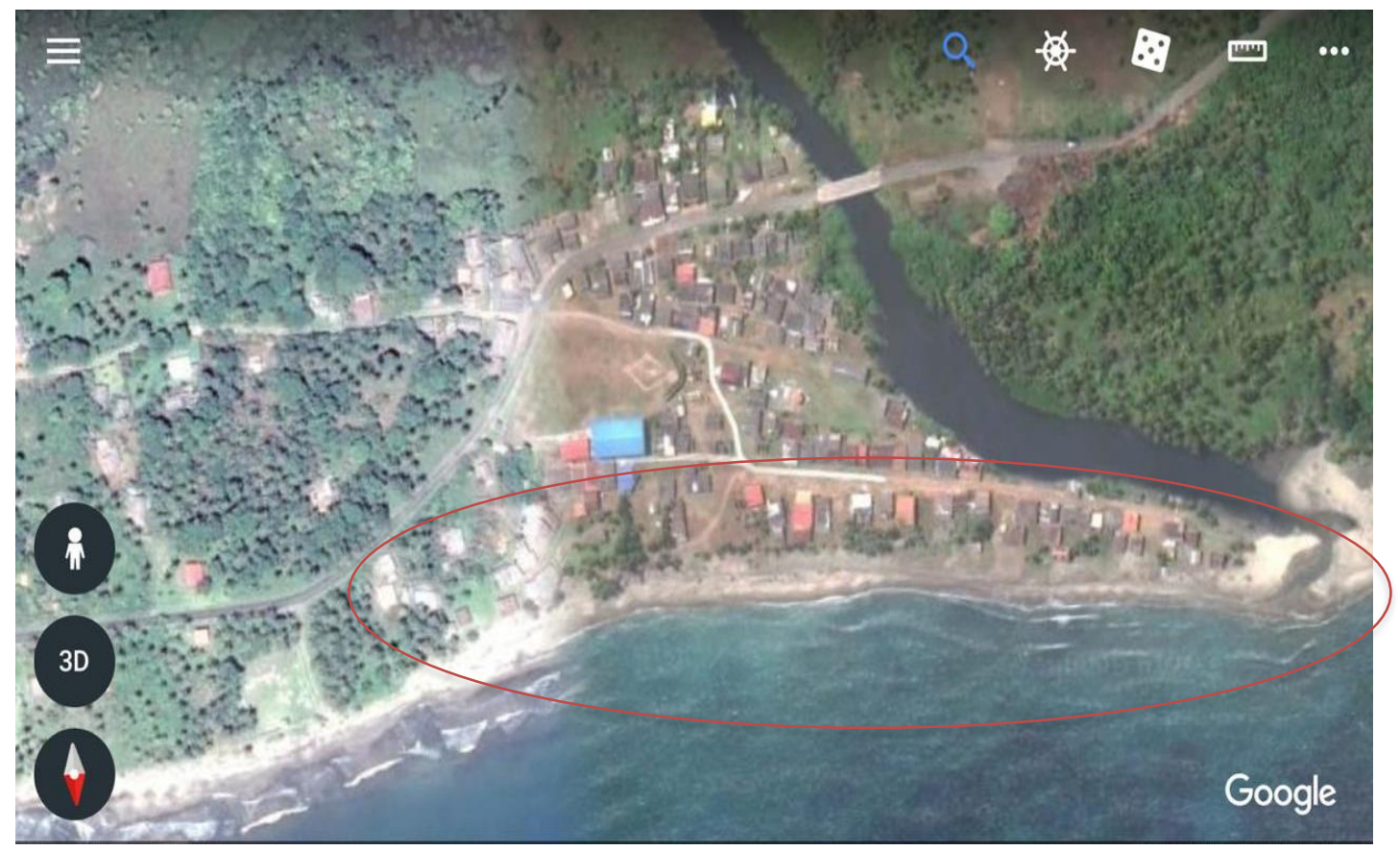

Figura 1: Ubicación geográfica de la Comunidad de Gobea, Distrito de Donoso, Provincia de Colón

Por su parte, el informe del Plan Estratégico Distrital del Municipio de Donoso (2017) señala que el área posee una biodiversidad variada, por sus ecorregiones, vegetación, bosques y especies animales. Los bosques brindan refugio a más de 650 especies de flora y fauna, la mayoría amenazadas, raras, endémicas regionales, binacionales y nacionales, de distribución restringida, y con poblaciones vulnerables y en peligro de extinción.

Respecto a la vida marina, en Donoso el arrecife sirve de albergue a peces, crustáceos, moluscos y muchas otras especies, que habitan formando grandes colonias. Dado el hecho que el talud continental se encuentra prácticamente al borde del arrecife, allí se producen especies de techos de vida, dependiendo de los niveles de profundidad. Estos arrecifes, además, hacen impracticable la pesca comercial de arrastre, ya que destruye las redes de los barcos de pesca. En cambio, estos "bajos costeros crean bancos muy atractivos para la pesca artesanal, bien sea a mano o con tramayos (Municipio de Donoso, 2017) 


\begin{tabular}{|c|c|c|}
\cline { 2 - 3 } & REVISTA SABERES APUDEP & Volumen 4 Número 1 \\
\hline
\end{tabular}

\section{MATERIALES Y MÉTODOS}

Para el desarrollo del estudio se realizaron cuatro (4) visitas de trabajo de campo, la primera (fase indagatoria) para la observación directa lo que permitió revisar y obtener información relevante de las áreas naturales existentes identificando los puntos críticos con mayor presencia de residuos sólidos, considerando aquellas que representaban un riesgo con posible contaminación ambiental y las tres (3) restantes, corresponden a la fase descriptiva. El trabajo de campo se organizó de la siguiente manera: una (1) en el mes de noviembre, 2018, otro (1) en el mes de diciembre 2018, y el último (1), se realizó en el mes de enero de 2019. Los muestreos se efectuaron por dos días seguidos, desde las 10 am a la 3pm, por día.

En la fase descriptiva (figura 2) se procedió a delimitar los sitios seleccionados en la franja costera, utilizando 2 transectos lineales, cada uno de $30 \mathrm{~m}$, separados por $10 \mathrm{~m}$ a lo largo de la playa de la comunidad en estudio. En cada cuadrante, se recolectaron los desechos sólidos los cuales fueron caracterizados de acuerdo al tipo de material (materia orgánica, plásticos, vidrios, latas, cartones, bloques cemento, entre otros). Esta operación se efectuó, con el fin de determinar cuáles son los residuos domiciliaros que más se vierten en las playas. Una vez clasificados los desechos sólidos, se depositaron en bolsas de plástico reciclable y previamente pesadas. Ya dentro de las bolsas fueron compactadas hasta no dejar espacio en el interior. Se procedió al pesaje y registro de cada bolsa por trabajo de campo, de modo que los datos pudieran ser analizados y presentados en tablas y gráficos.
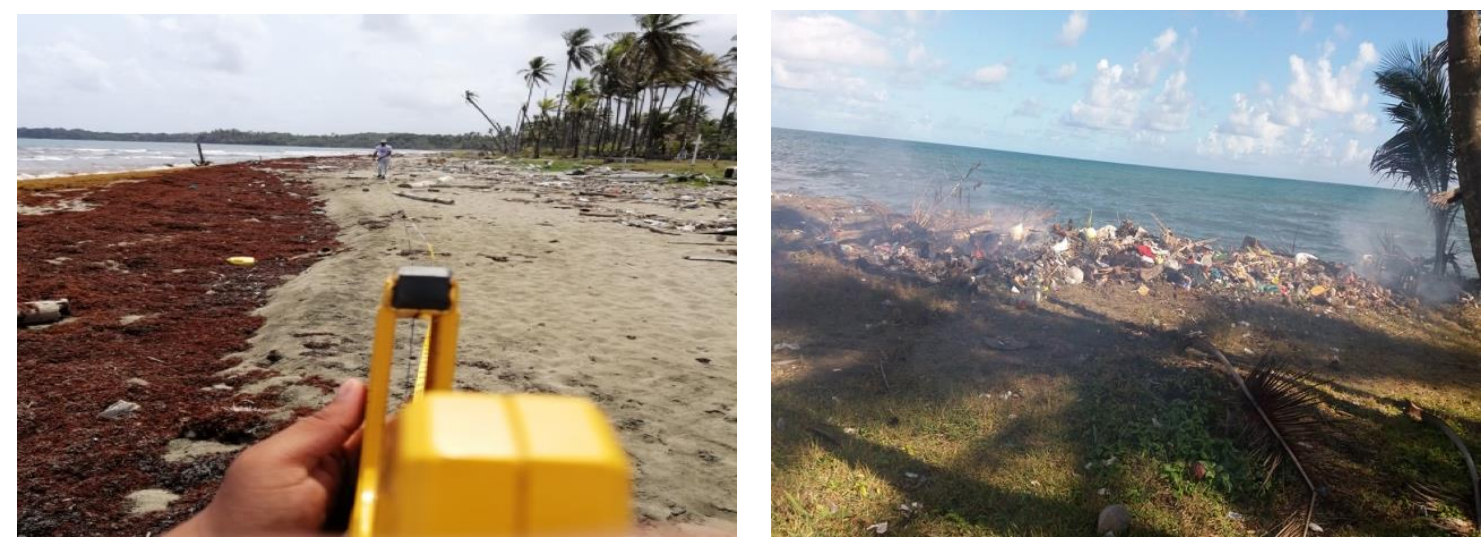

Figura 2. Preparación de área de estudio para la ubicación de los transectos 


\begin{tabular}{|c|c|c|}
\cline { 2 - 3 } & REVISTA SABERES APUDEP & Volumen 4 Número 1 \\
\hline
\end{tabular}

\section{RESULTADOS Y DISCUSIÓN}

La cantidad de desechos colectados en los tres muestreos realizados, demuestran un aumento progresivo en la variedad y cantidad de materiales como el plástico, el vidrio, latas, vegetales, hierbas, bloques de cemento, madera y restos de material de origen vegetal.

En el primer muestreo de la fase descriptiva se recolectaron un total de $163.5 \mathrm{Kg}$ de residuos sólidos, distribuidos en inorgánicos lo que representa un 95\% del peso y orgánicos de 5\%. Los resultados de este muestreo (Figura 3), presentan la variedad de residuos sólidos, mostrando la mayor proporción los inorgánicos de la siguiente manera: el plástico (39\%), vidrios (26\%), y aluminio (15\%), por su parte el caucho, telas, papel, porcelana suman un $16 \%$, mientras que los de materia orgánica y de origen vegetal ocupan un $4 \%$.

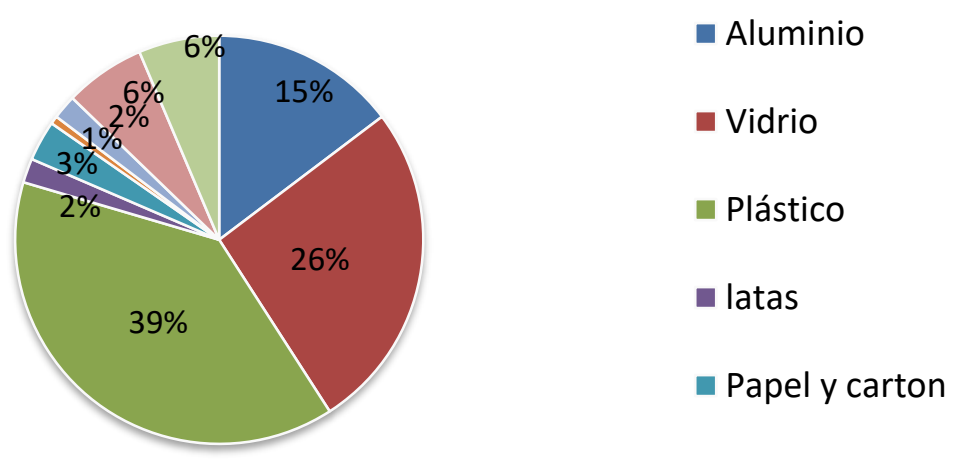

Figura 3. Transecto 1, Variedad de desechos sólidos inorgánicos encontrados

El segundo muestreo de la fase descriptiva evidencia la variedad de residuos sólidos encontrados, está vez el peso registrado fue $1,369.9 \mathrm{~kg}$, mostrando un $76 \%$ del peso total de residuos inorgánicos tal es el caso del plástico con un $37 \%$, vidrios con un $25 \%$, aluminio con un $14 \%$, y el resto de los residuos registrados representan un $24 \%$. Es importante señalar que la presencia de los bloques de cemento, los cuales generan un incremento es posible que se deba a los fenómenos naturales que se dieron en la comunidad, afectando un número considerable de viviendas, dejándolas totalmente destruidas (Figura 4). 
REVISTA SABERES APUDEP
ISSN L 2644-3805

Acceso Abierto. Disponible en:

https://revistas.up.ac.pa/index.php/saberes_apudep
Volumen 4 Número 1

Enero-Junio 2020

Recibido: 22/10/20; Aceptado: 26/12/20

pp. 68-83
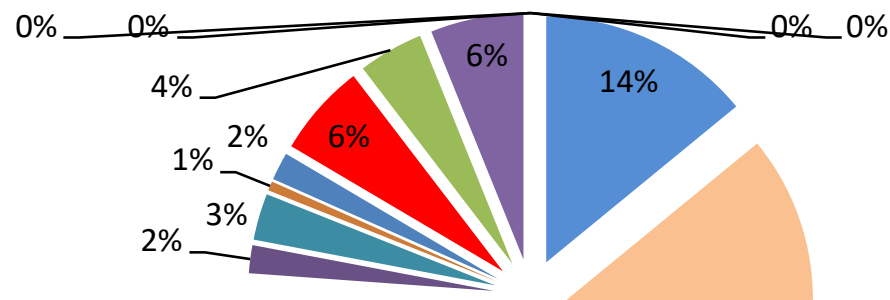

$25 \%$

$37 \%$

Aluminio
- latas
Porcelana
Madera.
Telas (Trapos)

Vidrio

- Papel y carton

- Caucho

Bloques de cemento.

Peso de la bolsa

\author{
Foam \\ - Vegetales hierbas cocos \\ Alambre de cerca. \\ Plástico \\ - Alambre de cerca.
}

figura 4. descripción de los desechos sólidos encontrados en el segundo muestreo

El tercer muestreo de la fase descriptiva se encontró $1033.5 \mathrm{~kg}$ de residuos sólidos orgánicos e inorgánicos, observándose que los de tipo inorgánico mantiene mayor proporción (65\%), siendo el plástico con un 34\% como el material con mayor presencia, el vidrio con un $12 \%$, y el resto de los residuos inorgánicos registrados suman un 19\%, por otro lado, el material orgánico de origen vegetal con un 35\%, (Figura 5). 


\begin{tabular}{|c|c|c|}
\cline { 2 - 3 } & REVISTA SABERES APUDEP & Volumen 4 Número 1 \\
\hline
\end{tabular}

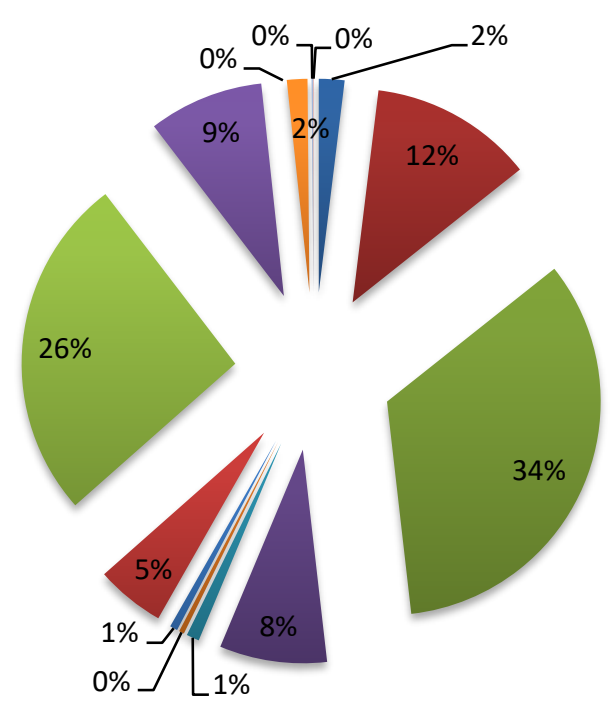

Aluminio
Vidrio
Plástico
latas
Papel y cartón
Foam
Porcelana
Caucho
Vegetales hierbas cocos
Madera.

Figura 5. Proporción de los residuos sólidos encontrados del tercer muestreo

Los resultados obtenidos en el estudio refuerzan el hecho de que la zona costera de la Comunidad de Gobea, ha sido utilizada por años como vertedero improvisado, y las razones conocidas por todos que los servicios de colecta de residuos sólidos no llegan hasta esta comunidad, lo que supone que sus pobladores recurren a la quema o la apertura de fosas en áreas naturales de la zona costera para deshacerse de los residuos, como posible evidencia de malas prácticas.

Los tres muestreos exponen una gran cantidad de material inorgánico acumulado, en comparación con los orgánicos, lo que supone una falta de manejo apropiado de los desechos sólidos de ese tipo. Uno de los desechos sólidos inorgánicos de mayor presencia corresponde al plástico, por su baja degradación se convierte en un problema mayor por su acumulación.

Llama la atención que en cada visita se reflejaba un incremento significativo de los residuos sólidos en mala disposición, lo que supone puede poner en riesgo la calidad ambiental del sitio, si no se actúa con responsabilidad y a tiempo. Cabe la posibilidad de que, al no presentarse alternativas amigables con el ambiente para minimizar la acumulación de residuos sólidos en mala disposición, puede empeorar la condición afectando grandemente el 


\section{REVISTA SABERES APUDEP \\ ISSN L 2644-3805}

Acceso Abierto. Disponible en:

https://revistas.up.ac.pa/index.php/saberes_apudep

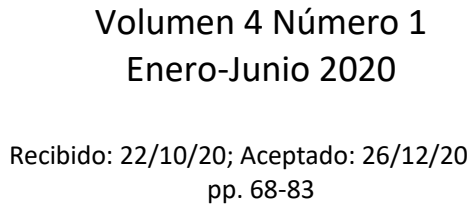

Recibido: 22/10/20; Aceptado: 26/12/20 pp. $68-83$

área. Tampoco se evidencia el uso útil de los residuos orgánicos de origen vegetal para revertir al suelo, desaprovechando sus beneficios para posibles actividades de agroforestería.

En esta comunidad costera inciden fenómenos naturales como es el caso de las mareas altas, torrenciales aguaceros, escorrentías entre otras, los cuales arrastran y acumulan en zona costera una gran cantidad de desechos sólidos depositados en la playa, de tipo orgánico como los de origen vegetal, aquellos restos de animales muertos, restos de palmeras y cocos, otros provenientes de cortes de hierbas, aquellos desechos sólidos producto de la actividad pesquera y los domiciliarios proveniente de la comunidad, los inorgánicos, los cuales no se biodegradan, dejándolos expuestos a cielo abierto, sin ningún manejo adecuado. De acuerdo a los resultados, los desechos sólidos que se encontraron en mayor proporción son los inorgánicos.

Por su parte, la presencia de materiales como bloques y cemento en los registros de los muestreos supone que producto de un mal tiempo en el área, siendo el resultado de una tormenta tropical, a finales del 2018 (María), afectando algunas casas de los moradores, echando abajo las construcciones. También generó afectaciones en el cementerio del lugar dejando expuestas algunas tumbas, donde el fuerte oleaje arrastró desechos sólidos como restos de los ataúdes. La comunidad fue afectada dejando un panorama preocupante no solo por el fenómeno natural, sino por las actividades antrópicas que generan desechos sólidos. Toda esta mezcla de grandes depósitos de desechos sólidos y el mar supone el deterioro de la calidad del suelo, las afectaciones de las corrientes subterráneas e inclusive aquellas especies marinas que son de consumo humano, lo que se presume puede afectar la salud humana y la salud ambiental del sitio.

En el segundo y tercer muestreos se vieron condicionados por lluvias intensas e inundaciones y la destrucción de 2 casas cercanas a la playa causadas por fuertes temporales. Algunos aportes de comentarios de los lugareños, señalan que, para el mes de diciembre, mientras la comunidad se prepara para las fiestas de fin de año, hay quienes vierten una gran cantidad de desechos sólidos domiciliarios provenientes de las compras navideñas, aumentando la mala disposición sin evidencias de manejo apropiado para éstos y agudizando la problemática. Sobre la base de las ideas expuestas y de los datos recabados en el estudio, 


\section{REVISTA SABERES APUDEP \\ ISSN L 2644-3805}

Acceso Abierto. Disponible en:

https://revistas.up.ac.pa/index.php/saberes_apudep

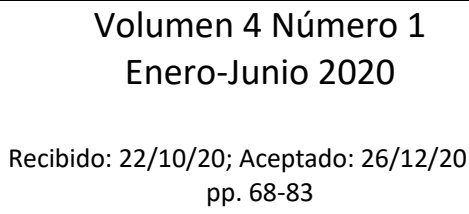

Recibido: 22/10/20; Aceptado: 26/12/20 pp. 68-83

se puede señalar que están creadas las condiciones en la Comunidad de Gobea para diseñar una propuesta piloto que vaya dirigida a minimizar la acumulación de los desechos sólidos generados y depositados en la zona costera, con acciones que van apoyadas en Educación ambiental y reciclaje. Todo esto puede ser en alianza y dirigido con líderes comunitarios y autoridades locales, que potencien al sitio como área turística, aprovechando las nuevas vías de acceso al lugar.

\section{CONCLUSIÓN}

Aunque existen aportes a la acumulación de desechos sólidos en la zona costera y áreas naturales de la Comunidad de Gobea por procesos y fenómenos naturales, las actividades antrópicas representan una de principales fuentes de contaminación ambiental, aportando a la degradación del paisaje natural de esta parte costera de la Provincia de Colón.

Toda esta mezcla de grandes depósitos de desechos sólidos (orgánicos e inorgánicos) con el mar supone el deterioro de la calidad del suelo, las afectaciones de las corrientes subterráneas e inclusive aquellas especies marinas que son de consumo humano, lo que da la posibilidad de afectar la salud humana y la salud ambiental del sitio.

En esta área costera, inciden fenómenos naturales como es el caso de las mareas altas, torrenciales aguaceros, escorrentías entre otras, los cuales arrastran y acumulan una gran cantidad de desechos sólidos depositados en la playa, de tipo orgánico como los de origen vegetal, aquellos restos de animales muertos, pencas, troncos, cocos, cortes de hierbas, aquellos producto de la actividad pesquera además de los domiciliarios proveniente de la comunidad, además de los inorgánicos los cuales no se biodegradan dejándolos expuestos a cielo abierto, mostrando un posible escenario de contaminación ambiental.

La generación y mala disposición de los residuos sólidos deben ser tema prioritario de las autoridades locales y líderes comunitarios de este distrito, que los oriente a organizar a fin lograr disminuir estos depósitos improvisados, garantizando la calidad ambiental de sus playas, mejorando y explotando la imagen del sitio como destino turístico con excelentes condiciones ambientales, aprovechando las nuevas vías de acceso a la costa abajo de la Provincia de Colón. Están dadas las condiciones para preparar un plan ajustado a la realidad 


\begin{tabular}{|c|c|c|}
\cline { 2 - 3 } & REVISTA SABERES APUDEP & Volumen 4 Número 1 \\
\hline
\end{tabular}

actual de la comunidad y todas las áreas vecinas. Para ellos se recomienda establecer el uso de la Educación ambiental y actividades de reciclaje, reforzadas con alianzas estratégicas que involucren a Mi Ambiente, Líderes comunitarios, autoridades locales, Universidad de Panamá, y voluntarios que vigilen la protección y mantenimiento del aseo y la urbanidad del lugar, asegurando la salud y la calidad ambiental.

\section{REFERENCIAS BIBLIOGRÁFICAS}

Alonzo M., E. R., \& Paz Hernández, C. (2014). Generación Y Manejo De Residuos Sólidos En Áreas Naturales Protegidas Y Zonas Costeras: El Caso De Isla Holbox, Quintana Roo. (5), 92-114. Disponible En: Https:/Www.Redalyc.Org/Articulo.Oa?ld=4557/455745079006

Arias, F.D. (25 De Septiembre De 2015), Océanos Abrumados Por Los Desechos, En Su Mayoría Plásticos. La Prensa. Recuperado Https://Www.Prensa.Com/Salud Y Ciencia/Oceanos-Playas-Costas-AbrumadosDesechos 0 4308319275. Html

Autoridad Nacional Del Ambiente De Panamá. (2012). Proyecto Integral Para El Desarrollo De La Costa Abajo De Colón (PIDCAC). Dirección De Gestión Integrada De Cuencas Hidrográficas (DIGICH) Y Fondo Mixto HispanoPanameño De Cooperación.

Banco Mundial BIRF- AIF (20 De Septiembre De 2018). Los Desechos: Un Análisis Actualizado Del Futuro De La Gestión De Los Desechos Sólidos. Https://Www.Bancomundial.Org/Es/News/Immersive-Story/2018/09/20/What-AWaste-An-Updated-Look-Into-The-Future-Of-Solid-Waste-Management

Blais, A., Berlin, R., Clima, R., Glendening, J., Gutierrez, V., Izard, J., Mallow,C., Nelson, R., Ricth, E., Swigert, P. (2010). El Manejo De Basura En Colón, Panamá:Un Estudio De Estrategias Seleccionadas. Universidad De Virginia 2010, Departamento De Las Ciencias Medioambientales, Departamento De La Política, Charlottesville.

Benseny, G. (2011), Tesis Doctoral: La Zona Costera Como Escenario Turístico. Transformaciones Territoriales En La Costa Atlántica Bonaerense Villa Gesell (Argentina), Universidad Nacional De Sur, Bahía Blanca. 


\begin{tabular}{|c|c|c|}
\cline { 2 - 3 } & REVISTA SABERES APUDEP & Volumen 4 Número 1 \\
\hline
\end{tabular}

Contreras S., M. J. (2008). Evaluación De Experiencias Locales Urbanas Desde El Concepto De Sostenibilidad: El Caso De Los Desechos Sólidos Del Municipio De Los Patios (Norte De Santander, Colombia). Trabajo Social (10), 109-134

Esquer, R. (2009). Reciclaje Y Tratamiento De Los Residuos Sólidos Urbanos. Instituto Polytechnic Nacional, México, D.F.

FEE - Foundation for Environmental Education (2010) Blue Flag Beach Criteria And Explanatory Notes - 2010-2011. Foundation Foro Enviro mental Educación, Copenhagen.

Hurtado, Y. (2010). Determinación De Un Modelo De Medición De Capacidad De Carga En Playas Turísticas De Uso Intensivo, Como Herramienta Para El Manejo Integrado Costero. Aplicación En La Playa El Rodadero (Santa Marta, Colombia). Tesis De Maestría En Manejo Integrado Costero, Universidad Del Magdalena. Santa Marta, Colombia.

Junco, R. \& Rodríguez, D. (2000). Desechos Hospitalarios: Aspectos Educativos En La Implementación De Su Manejo. Revista Cubana Higiene Y Epidemiología, 38(3), 195-200.

Kofalusi, G. \& Aguilar, G. (2006). Los Productos Y Los Impactos De La Descomposición De Residuos Sólidos Urbanos En Los Sitios De Disposición Final. Gaceta Ecológica 79. Instituto Nacional De Ecología, México; 39-51

Macfadyen, G.; Huntington, T.; Cappell, R. (2011), Aparejos De Pesca Abandonados, Perdidos O Descartados. Informes Y Estudios Del Programa De Mares Regionales, PNUMA N.O 185; FAO Documento Técnico De Pesca Y Acuicultura N.O 523. Roma, PNUMA/FAO. 2011.

Márquez Gulloso, E., Rosado Vega, J.R. (2011). Clasificación E Impacto Ambiental De Los Desechos Sólidos, Generados En Las Playas De Riohacha, La Guajira Colombia. Universidad De La Guajira, Colombia. Revista Facultad De Ingeniería. Universidad De Antioquía (60); 118-128.

Mazzeo, N. E. (2012) Manual Para La Sensibilización Comunitaria Y Educación Ambiental: Gestión Integral De Residuos Sólidos Urbanos. - 1a Ed. - San Martín: Inst. Nacional De Tecnología Industrial - Inti, 2012. E-Book.

Municipio De Donoso. (2017), Plan estratégico distrital 2018-2022, Panamá, República De Panamá. 


\begin{tabular}{|c|c|c|}
\cline { 2 - 3 } & REVISTA SABERES APUDEP & Volumen 4 Número 1 \\
\hline
\end{tabular}

Ojeda, S., Lozano, G., Quintero, M., Whitty, K., Smith, C. (2008). Generación De Residuos Sólidos Domiciliarios Por Periodo Estacional: El Caso De Una Ciudad Mexicana. I Simposio Iberoamericano De Ingeniería De Residuos. Castellón, España.

Pereira, G. \& Conto, S. (2008). Manejo De Desechos Sólidos En Un Evento Turístico, Fiesta Nacional De La Uva. Universidad De Caxias Do Soul, Brasil.

Rodríguez, G. A., B. L. Toro, \& G. J. H. (2008). Ciudades Ambientalmente Sostenibles. Universidad Del Rosario.

Sáez, A. \& Urdaneta, J. (2014), Manejo De Residuos Sólidos En América Latina Y EI Caribe. Revista OMNIA, 20(3),121-135

Silgado R, J. (2006). La Gestión De Residuos Sólidos Urbanos En La Ciudad Histórica Y Sostenible: El Ejemplo De Andalucía. Segundas Jornadas Sobre Investigación En Arquitectura Y Urbanismo. Sevilla

Universidad De Panamá (2010), Ecología Y Ambiente, Disponible En:

Https://Vicinvestigacion.Up.Ac.Pa/Sites/Vicinvestigacion/Files/Publicaciones/Libros/E colog\%C3\%Ada-Ambiente-97-134.Pdf

Valencia O., V. M. (2009). Diseño E Implementación Del Plan De Manejo Integral De Residuos Solidos De La Corporación Club Campestre - Medellín - Llanogrande. Corporación Universitaria La Sallista. Facultad De Ingeniería. Caldas, Medellín, Colombia. 